\title{
Improvement of Lax Skin Using a High-Pressure Pneumatic Dermal Delivery System
}

\author{
Ik Jun Moon', Ji Su Yu² \\ Won Ah $\mathrm{Choi}^{3}$, Tai Kyung Noh ${ }^{1}$ \\ Woo Jin Lee', Sung Eun Chang ${ }^{1}$ \\ Mi Woo Lee ${ }^{1}$, Jee Ho Choi ${ }^{1}$ \\ Chong Hyun Won ${ }^{1 *}$, Jiehoon Kim*
}

\author{
${ }^{1}$ Department of Dermatology, Asan Medical \\ Center, University of Ulsan College of Medicine, \\ Seoul, Korea \\ ${ }^{2}$ College of Medicine, University of Toledo, Ohio, \\ USA \\ ${ }^{3}$ Asan Institute for Life Sciences, Seoul, Korea \\ ${ }^{4}$ Dr. Kim's Skin \& Laser Clinic, Suwon, Korea
}

Received December 13, 2016

Revised December 16, 2016

Accepted December 16, 2016

\author{
Correspondence \\ Chong Hyun Won \\ Department of Dermatology, Asan Medical \\ Center, University of Ulsan College of Medicine, \\ 88 Olympic-ro 43 gil, Songpa-gu, Seoul 05505 , \\ Korea \\ Tel.: +82-2-3010-3460 \\ Fax: +82-2-486-7831 \\ E-mail: drwondamc.seoul.kr \\ Jiehoon Kim \\ Dr. Kim's Skin \& Laser Clinic, Paldal-gu, \\ Kwonkwang-ro 197, Suwon 16489, Korea \\ Tel.: +82-31-222-7576 \\ Fax: +82-31-222-7583 \\ E-mail: jiehoonđanaver.com \\ *These two authors contributed equally to this \\ work as corresponding authors. \\ (C) Korean Society for Laser Medicine and Surgery \\ (@) This is an open access article distributed under the \\ terms of the Creative Commons Attribution Non- \\ Commercial License (http://creativecommons.org/ \\ licenses/by-nc/4.0) which permits unrestricted non- \\ commercial use, distribution, and reproduction in any \\ medium, provided the original work is properly cited.
}

\section{Background and Objectives}

The high-pressure pneumatic delivery system utilizes an ejection of highpressure gas delivering desired substances into the subcutaneous tissue and subcutaneous musculoaponeurotic layer.

\section{Materials and Methods}

To assess the efficacy and safety of the system, a split-face study was performed. We enrolled18 Asian subjects. Multiple 20\%-glucose injections were administered to the temporal area using the highpressure pneumatic delivery system. Clinical photographs were obtained at baseline and 2 weeks, 1 month, 2 months, and 3 months after injections. A five-point grading scale was used by to evaluate the results. To evaluate the histological effect of the high-pressure pneumatic delivery system, specimens treated with various injection pressures were examined histologically.

\section{Results}

Subjective and objective assessments of skin laxity showed significant improvements 3 months after the treatment. Immediately after the procedure, the treated area showed noticeable tissue augmentation. No significant procedure-related adverse reactions were observed. Study limitations were the inclusion of relatively young-aged subjects and a small number of male subjects. Histologically, the specimens treated with the high-pressure pneumatic delivery system demonstrated numerous dermal vesicles composed of the injected fluid, which were located deeper in the dermis when a higher injection pressure was applied.

\section{Conclusion}

A high-pressure pneumatic delivery system proved to be safe and effective for skin laxity correction and resultant nasolabial folds in Asians.

\section{Key words}

Face lifting; Needle-free injector; Needleless microjet device; Pressure 


\section{INTRODUCTION}

Skin wrinkles result from various aging processes, with decreased skin laxity being the most common causative factor. Multiple techniques to improve lax skin, including facelifts and radiofrequency therapy, have shown varying degrees of success and associated side effects. Essentially, facelifts are considered to provide the best correction of facial rhytides. ${ }^{1}$ However, despite its dramatic effects, facelifts require excessive pain control during procedure and a long recovery time before any improvements are seen. ${ }^{2,3}$ Conversely, noninvasive modalities such as ultrasound and radiofrequency therapies have the advantages of short downtimes and relatively low risk profiles, but usually require multiple treatment sessions to be effective. ${ }^{3}$ Thus, treatment modalities that result in regeneration of the dermal matrix and consequently improve skin laxity with minimal risk and downtime are highly sought after. Recently, dermal drug delivery systems that use high pneumatic pressure have been created to inject various drugs into the dermis without using a needle. ${ }^{4.5}$ In addition to the intended dermal drug delivery, tissue augmentation with an increased firmness were observed. ${ }^{6-9}$ This fortuitous finding, thought to be the result of dermal fibrosis, led to the idea of using high-pressure pneumatic dermal drug delivery systems to improve skin turgor. We hereby introduce a clinical trial evaluating the efficacy and safety of a novel high-pressure pneumatic dermal delivery system for the improvement of lax skin and resulting

A

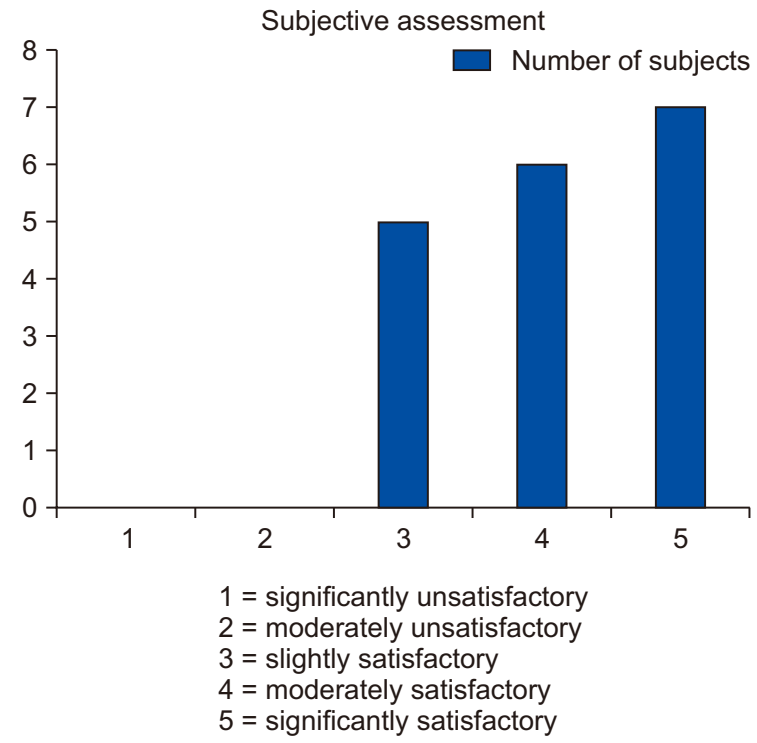

nasolabial folds.

\section{MATERIALS AND METHODS}

\section{Patients and Treatment Protocol}

Subjects enrolled in this clinical study were volunteers among patients who visited a private dermatology clinic from October 2015 to January 2016. Subjects aged $>20$ years who complained of skin laxity were considered eligible. Potential subjects were excluded if they (1) had received facelifts or any other surgical procedure in the intended treatment area; (2) had a history of subcutaneous infections in the area within the previous 4 weeks; (3) had a significant psychiatric problem such as body dysmorphia; (4) had taken aspirin within the previous 2 weeks; or (5) were pregnant or lactating. Subjects were treated with a high-pressure pneumatic delivery system (AIRJET ${ }^{\circledR}$; UNION MEDICAL CO., LTD., Uijeongbu, Koreal uniformly according to the following protocol: 40 shots around the supra-auricular area using a pneumatic pressure of 3.5 bar with $77 \mathrm{~ms}$ injection duration of $20 \%$ glucose. Neither local anesthesia nor post-treatment dressing were required. A 5-minute gauze compression was used for hemostasis.

\section{Evaluation of clinical efficacy and safety}

An evaluator-blinded split-face trial was carried out by photographic evaluation with the treatment side chosen randomly. Baseline clinical photographs were obtained

B

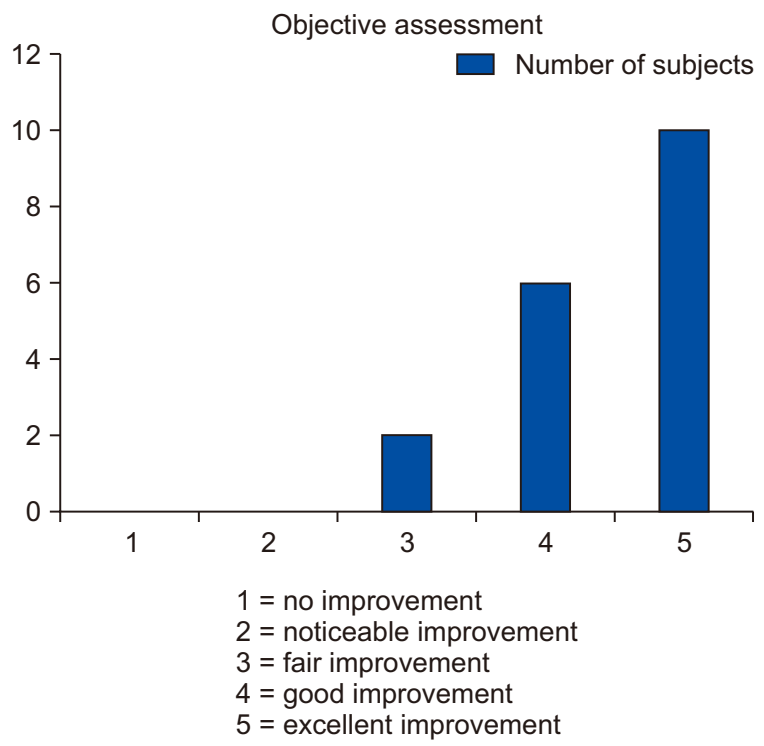

Fig. 1. Improvement in (A) subjective assessment scores and (B) objective assessment scores evaluated before and at 3 months after treatment with the high-pressure delivery system. 
before the treatment. All subjects were requested to visit the clinic for assessment and clinical photographs 2 weeks, and 1, 2, and 3 months after the procedure.

The results of the treatment were analyzed using both subjective and objective evaluations by the patients and the physician. Objective improvement was assessed by two independent and experienced dermatologists using clinical photographs after the final session and during the 3 months thereafter on a 5-point grading scale defined as follows: 1 = no improvement, 2 = noticeable improvement, 3 = fair improvement, 4 = good improvement, 5 = excellent improvement. The data collected at baseline and 3 months after treatment were compared for final evaluation of clinical efficacy.

The subjective assessment was obtained with a questionnaire composed of a 5-point grading scale defined as follows: 1 = significantly unsatisfactory, 2 = moderately unsatisfactory, 3 = slightly satisfactory, 4 = moderately satisfactory, 5 = significantly satisfactory. Additionally, to assess the risk of potential discomfort or long-lasting symptoms related to the procedure, a questionnairebased survey was performed at the last follow-up visit.

\section{Histological evaluation}

A skin specimen of size $2 \times 2 \mathrm{~cm}$ was retrieved from a tissue that would otherwise be discarded during an operation. Injections with a high-pressure pneumatic delivery system were carried out using two different pneumatic pressures: 2.3 bar and 4.6 bar. The duration of injection was kept constant at $77 \mathrm{~ms}$. The specimens were immediately sent to the Department of Pathology following the injections with a high-pressure pneumatic delivery system.

\section{Statistical analysis}

Statistical analysis was performed using SPSS version 19.0 for Windows (Statistical Package for the Social Sciences, SPSS, Chicago, USA). The paired t-test was used for evaluation of statistical significance between the score of both objective and subjective assessments at baseline and 3 months after treatment. Differences were considered statistically significant when $p$ values were $<0.05$.

\section{RESULTS}

\section{Evaluation of clinical efficacy and safety}

Initially, 23 patients volunteered to participate in the study; however, 5 patients were excluded from the study because they were lost to follow-up. The reasons for this were personal, and not related to the procedure Isuch as
Table 1. Individual demographic data of the volunteers with respective subjective and objective assessment scores evaluated 3 months after treatment.

\begin{tabular}{ccccc}
\hline $\begin{array}{c}\text { Patient } \\
\text { No. }\end{array}$ & Sex & Age & $\begin{array}{c}\text { Subjective } \\
\text { Assessment Score }\end{array}$ & $\begin{array}{c}\text { Objective } \\
\text { Assessment Score }\end{array}$ \\
\hline 1 & $\mathrm{~F}$ & 27 & 3 & 4 \\
2 & $\mathrm{~F}$ & 45 & 5 & 4 \\
3 & $\mathrm{~F}$ & 48 & 3 & 4 \\
4 & $\mathrm{~F}$ & 39 & 5 & 5 \\
5 & $\mathrm{M}$ & 35 & 5 & 5 \\
6 & $\mathrm{~F}$ & 51 & 4 & 4 \\
7 & $\mathrm{~F}$ & 31 & 5 & 5 \\
8 & $\mathrm{~F}$ & 37 & 4 & 4 \\
9 & $\mathrm{~F}$ & 41 & 4 & 4 \\
10 & $\mathrm{M}$ & 41 & 5 & 5 \\
11 & $\mathrm{~F}$ & 38 & 3 & 4 \\
12 & $\mathrm{~F}$ & 25 & 3 & 3 \\
13 & $\mathrm{~F}$ & 30 & 4 & 4 \\
14 & $\mathrm{~F}$ & 43 & 4 & 3 \\
15 & $\mathrm{~F}$ & 38 & 5 & 5 \\
16 & $\mathrm{~F}$ & 25 & 4 & 4 \\
17 & $\mathrm{~F}$ & 48 & 3 & 4 \\
18 & $\mathrm{~F}$ & 43 & 5 & 1 \\
Mean score & & 4.22 & 4.11 \\
\hline
\end{tabular}

unsatisfactory outcomes or complications).

A total of 18 patients ( 2 men and 16 women) fulfilled all the requirements for the investigation. The mean age was 37.9 years, with a range of 25-51 years. Patient information and assessment outcomes are listed in Table 1. Preand post-treatment clinical photographs of one patient are shown as Fig. 2. Results of both objective and subjective assessments showed significant improvements. The average objective assessment score was 4.22, which indicated a good to excellent improvement in skin laxity. The average subjective assessment score was 4.11, implying good satisfaction. None of the patients reported longlasting discomfort or adverse reactions related to the procedure on the questionnaire.

\section{Histological evaluation}

Light microscopy evaluation of the skin specimen after treatment with a high-pressure pneumatic delivery system revealed significant histological alterations of varying degrees according to the parameter applied. Under a relatively low pressure of 2.3 bar, prominent vacuoles were observed in the papillary dermis (Fig. 3). Besides the vacuoles, spaces between the collagen fibers were enlarged. Treatment using a higher pressure of 4.6 bar resulted in even more numerous vacuoles and prominent widening of spaces between collagen fibers through the entire dermis. No apparent histological alteration was 

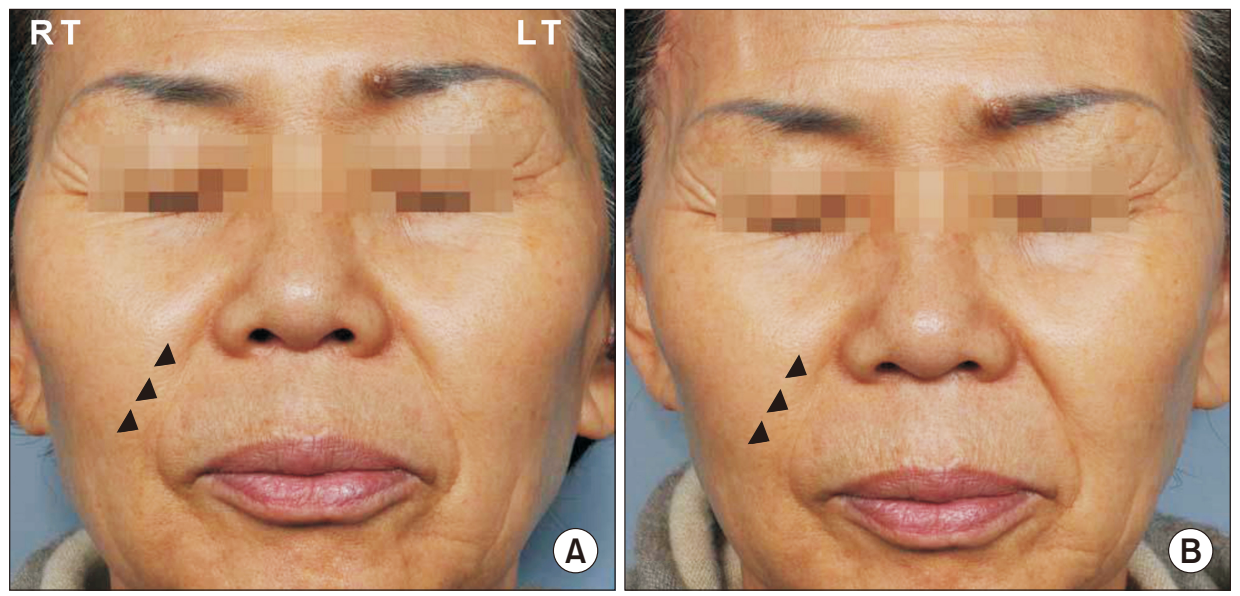

Fig. 2. Clinical photographs of patient number 2 (A) before the treatment and (B) 1 month after the treatment on her right supra-auricular area using the high-pressure delivery system. Note the improvement of her right nasolabial fold indicated by arrowheads.
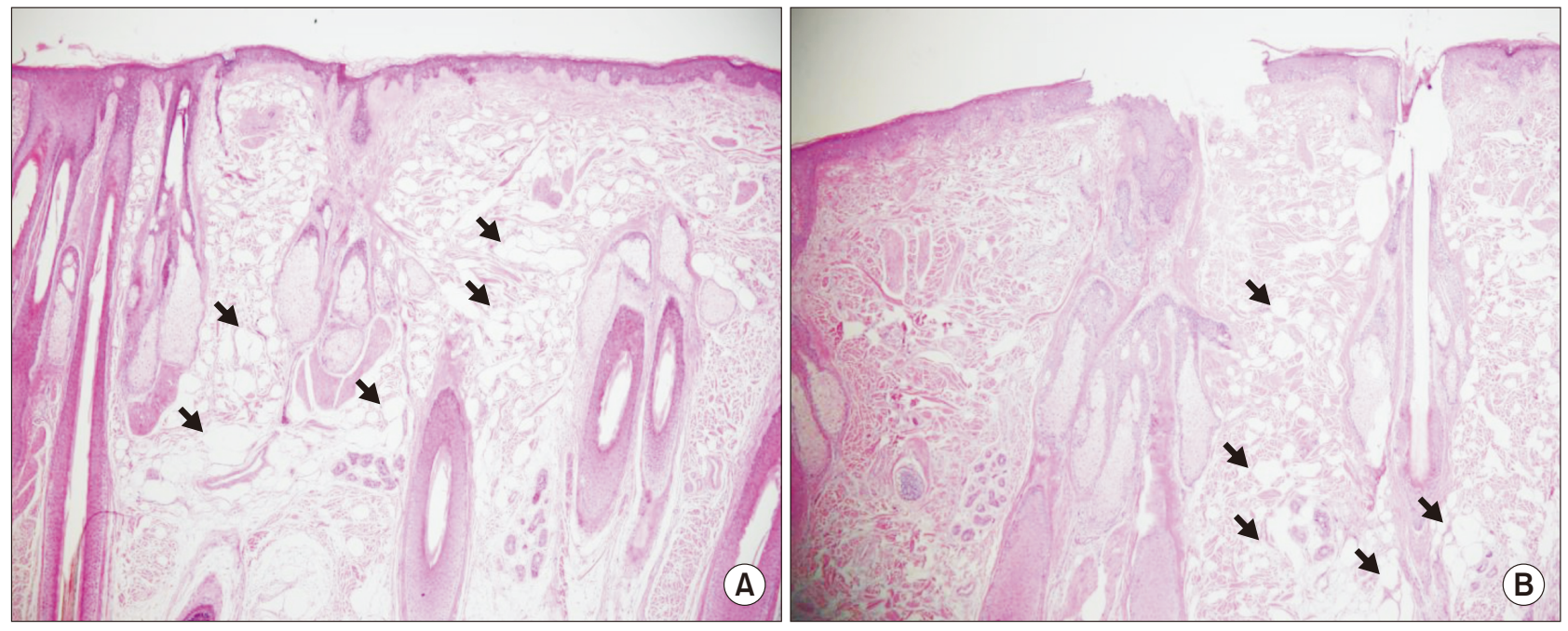

Fig. 3. Histological features following injection using the high-pressure delivery system. Vacuoles that consist of the delivered fluid are indicated by arrows. (A) Under relatively low pressure (2.3 bar), prominent vacuoles splitting the collagen fibers are mostly prominent superficially in the dermis. (B) A higher pressure (4.6 bar) results in numerous vacuoles and prominent widening of spaces between collagen fibers in the deep dermis.

noticeable in the subcutaneous fatty layer.

\section{DISCUSSION}

Correction of the inevitable aging of skin has been a challenge for dermatologists as well as cosmetic surgeons. Aging of skin encompasses a variety of processes including dermis thinning, tissue elasticity loss, and wrinkle formation. Restoration of skin laxity has been attempted for a long period as it may result in the correction of wrinkles and improved skin texture. Among various therapeutic options, atmospheric gas injection by means of high-pressure pneumatic needle-free injectors has proved to be effective in the treatment of scarring as well as in skin remodeling and the improvement of lax skin.

Skin laxity and resulting rhytides are known to be a consequence of decreased collagen content in the superficial dermis, and laxity of the deeply located subcutaneous musculoaponeurotic system (SMAS). Accordingly, the correction of lax skin can be achieved by remodeling both superficial and deep layers of the skin. In the present study, a high-pressure pneumatic delivery system histologically demonstrated its ability to target both superficial and deep dermis depending on the applied pressure; a finding consistent with a previously reported cadaveric study. ${ }^{10}$ In that sense, a high-pressure pneumatic delivery system not only targets the dermis, where it delivers both pneumatic force and liquid agents, but can also potentially stimulate SMAS at a higher pressure, causing fibrosis and shrinkage that ultimately lead to increased skin turgor.

A high-pressure pneumatic delivery system proved to 
be reliable, with increased subjective and objective assessment scores. Although they are effective in the treatment of lax skin, some therapeutic strategies lespecially surgical ones) cause treatment-related pain and discomfort and have long recovery periods. Thus, true 'satisfaction' of the patient is not guaranteed. The high satisfaction score in this study confirms that AIRJET is both an effective and gentle apparatus to be used in the field of cosmetic dermatology. The treatment protocol was simple, without the need for anesthesia, and the treatment session durations were short.

Regarding the safety profile, AIRJET did not produce any serious, long-lasting side effects. Transient headache, which subsided the next day without any treatment, was reported by two subjects. Local edema and erythema on the treated area lasting for a couple of hours was reported but did not lead to the formation of persistent erythema or post-inflammatory hyperpigmentation.

It is noteworthy that the use of solutions with high osmolarity (e.g., mannitol) has been demonstrated to enhance the immediate volume effect. The needle-free, pneumatic pressure-assisted injection could be applied using different solutions, depending on the desired effect. Accordingly, further investigations using other injectable agents may reveal effectiveness in treating various cutaneous conditions.

Limitations of this study include the small number of subjects, the low proportion of male subjects, and the lack of long-term follow-up to assess the duration of the therapeutic effects. Future investigations with a larger number of subjects to confirm the efficacy and safety of this novel technique are necessary. Furthermore, no consideration for the degree of photoaging or skin thickness and phototype was given when selecting the patients. In fact, the actual volume of fluid delivered depends largely on individual factors such as skin turgor or thickness even when using the same parameters. Hence, this variability requires the clinician's experience and skills to achieve optimal results. Another limitation of this study is that the evaluation of skin turgor and the degree of nasolabial folds mainly relied on subjective scoring. To enhance objectivity, an additional study using a well-established, universally accepted grading system for skin turgor and nasolabial folds could be of great value.

Augmentation of skin elasticity through dermal remodeling has been long been studied. Through the safe and effective correction of skin laxity demonstrated by AIRJET, it could be a powerful therapeutic option in the improvement of lax skin and resulting nasolabial folds.

\section{REFERENCES}

1. Botti C, Botti G. Facelift 2015. Facial Plast Surg 2015;31:491503.

2. Araújo AR, Soares VP, Silva FS, Moreira Tda S. Radiofrequency for the treatment of skin laxity: mith or truth. An Bras Dermatol 2015;90:707-21.

3. Suh DH, Lee SJ, Ryou JH, Son HC, Kim HJ, Kim HS. Monopolar radiofrequency treatment in Asian skin: do multiple RF treatments over time have beneficial effects? An observational report with long-term follow-up in eight patients. Dermatol Surg 2013;39:670-2.

4. Ravi AD, Sadhna D, Nagpaal D, Chawla L. Needle free injection technology: a complete insight. Int J Pharm Investig 2015;5:192-9.

5. Tagawa Y, Oudalov N, El Ghalbzouri A, Sun C, Lohse D. Needlefree injection into skin and soft matter with highly focused microjets. Lab Chip 2013;13:1357-63.

6. Kobus KF, Dydymski T. Quantitative dermal measurements following treatment with AirGent. Aesthet Surg J 2010;30:7259.

7. Kim BJ, Yoo KH, Kim MN. Successful treatment of depressed scars of the forehead secondary to herpes zoster using subdermal minimal surgery technology. Dermatol Surg 2009; 35:1439-40.

8. Lee JW, Kim BJ, Kim MN, Lee CK. Treatment of acne scars using subdermal minimal surgery technology. Dermatol Surg 2010;36:1281-7.

9. Levenberg A, Halachmi S, Arad-Cohen A, Ad-El D, Cassuto D, Lapidoth M. Clinical results of skin remodeling using a novel pneumatic technology. Int J Dermatol 2010;49:1432-9.

10. Seok J, Oh CT, Kwon HJ, Kwon TR, Choi EJ, Choi SY, et al. Investigating skin penetration depth and shape following needle-free injection at different pressures: a cadaveric study. Lasers Surg Med 2016;48:624-8. 